\title{
Does Diaphragmatic Electrical Activity in Preterm Infants Predict Extubation Success?
}

\author{
Neetu Singh MD MPH, Matthew J McNally RRT, and Robert A Darnall MD
}

\begin{abstract}
BACKGROUND: Despite many advances in respiratory care and mechanical ventilation, neonatologists lack an objective tool to aid in decision making for timely extubation. Electrical activity of the diaphragm $\left(\mathbf{E A _ { \mathrm { di } }}\right)$, a measure of neural respiratory drive and inspiratory load, may be a useful predictor of extubation success in preterm neonates. The objective of this work was to investigate whether peak $\mathbf{E A}_{\mathrm{di}}$ could distinguish successful versus failed extubation in mechanically ventilated preterm infants. METHODS: We examined peak $\mathbf{E A}_{\mathrm{di}}$ as a predictor of extubation outcome in a convenience sample of 21 preterm infants with respiratory distress syndrome requiring mechanical ventilation. Infants were ventilated with a VN500 ventilator using volume guarantee mode and extubated per unit protocol. Peak $\mathbf{E A}_{\mathrm{di}}$ was continuously measured with an $\mathbf{E A}_{\mathrm{di}}$ catheter in the esophagus to obtain data at 1-min intervals for $24 \mathrm{~h}$ before extubation. The primary outcome was extubation success, defined as not requiring re-intubation for at least $72 \mathrm{~h}$. RESULTS: Twenty one premature infants with respiratory distress syndrome included in the study had a mean \pm SD) gestational age of $28 \pm 2.6$ weeks and mean birthweight of $1,208 \pm 611 \mathrm{~g}$. The first extubation attempt occurred at a median age of $1 \mathrm{~d}$ (interquartile range 1-2 d). One third of the infants failed the first extubation attempt. Infants with failed extubations were significantly smaller (mean \pm SD birthweight $788 \pm 266 \mathrm{~g}$ versus $1,417 \pm 632 \mathrm{~g}$ ) and of lower gestational age (mean \pm SD $26 \pm 1.9$ weeks versus $29 \pm 2.6$ weeks) compared with those with successful extubation. Mean peak $\mathbf{E A}_{\mathrm{di}}$ before extubation did not differ between the 2 groups. CONCLUSIONS: This small study suggests that the pre-extubation peak $\mathbf{E} \mathbf{A}_{\mathrm{di}}$ does not predict extubation success. (ClinicalTrials.gov registration NCT02144363.) Key words: diaphragmatic electrical activity; extubation; preterm. [Respir Care 2018;63(2):203-207. (c) 2018 Daedalus Enterprises]
\end{abstract}

\section{Introduction}

Early extubation is important to prevent ventilator-induced lung injury and the long-term consequences of prolonged mechanical ventilation in preterm infants. ${ }^{1,2}$ No

\footnotetext{
Dr Singh is affiliated with the Department of Pediatrics, Mr McNally is affiliated with the Department of Respiratory Therapy, and Dr Darnall is affiliated with the Department of Neonatology, Dartmouth-Hitchcock Medical Center, Lebanon, New Hampshire.

Dr Singh presented a version of this work at the annual meeting of the Pediatric Academic Societies, held April 30 to May 3, 2016, in Baltimore, Maryland.

This work was supported by the Hearst Foundation, Dartmouth Hitchcock Medical Center, Lebanon, New Hampshire. Technical support and supplies were donated by MAQUET. The authors have disclosed no conflicts of interest.
}

universally accepted and validated measure exists to determine readiness for extubation. ${ }^{3}$ Instead, decisions to extubate are often made subjectively based on clinical judgment, leading to extreme variations in extubation practice and complications such as extubation failures, repeated intubations, and associated risk for airway injury and atelectrauma. ${ }^{4-8}$

Neural respiratory drive and the ability of the diaphragmatic muscle to respond to inspiratory load are the major determinants of extubation success in neonates. ${ }^{4,8-11}$ The electrical activity of the diaphragm $\left(\mathrm{EA}_{\mathrm{di}}\right)$, controlled by

\footnotetext{
Correspondence: Neetu Singh MD MPH, Division of Neonatology, Department of Pediatrics, Dartmouth Geisel School of Medicine, Dartmouth Hitchcock Medical Center, 1 Medical Center Drive, Lebanon NH 03756. E-mail: neetu.singh@hitchcock.org.
}

DOI: $10.4187 /$ respcare.05539 
brainstem respiratory neural networks, measures the neural respiratory drive to the diaphragm in proportion to the inspiratory load. ${ }^{12-14}$ Few studies have used $\mathrm{EA}_{\mathrm{di}}$ as a tool to predict the results of a spontaneous breathing test (SBT) in children and adults. ${ }^{10,15-17}$ However, $\mathrm{EA}_{\mathrm{di}}$ as an extubation predictor has not been studied in preterm infants with immature lungs, a group often exposed to mechanical ventilation and at great risk for ventilator-induced lung injury. We examined the association between pre-extubation peak $\mathrm{EA}_{\mathrm{di}}$, which represents neural respiratory effort on inspiration, and extubation outcome. We hypothesized that in mechanically ventilated preterm infants with respiratory distress syndrome (RDS), mean peak $\mathrm{EA}_{\mathrm{di}}$ values would be significantly different in infants with successful extubation compared with peak $\mathrm{EA}_{\mathrm{di}}$ values in those who failed extubation.

\section{Methods}

The study was conducted in a 30-bed neonatal ICU at the Dartmouth Hitchcock Medical center in Lebanon, New Hampshire. The Dartmouth College Committee for the Protection of Human Subjects approved and monitored the study. Parents of all included subjects gave informed consent.

Participants. A convenience sample of 21 preterm infants (born at $<35$ week gestation) with RDS requiring mechanical ventilation in the first $24 \mathrm{~h}$ after birth were recruited between June 2014 and December 2015. Infants with a non-intact esophagus (eg, tracheal-esophageal fistula or atresia), a non-functional diaphragm (eg, phrenic nerve palsy), severe intracranial hemorrhage or structural CNS abnormality, severe birth asphyxia, or critical illness requiring paralysis or deep sedation were excluded.

Procedures. All included infants were managed per unit protocol. They were initially stabilized receiving CPAP in the delivery room and only intubated in the first $24 \mathrm{~h}$ of life for worsening respiratory failure. Intubated infants were placed on a VN500 ventilator (Dräger, Telford, Pennsylvania) on assist control and volume guarantee mode. The ventilator parameters were set and adjusted by the primary care team as per unit guidelines. The initial tidal volume $\left(\mathrm{V}_{\mathrm{T}}\right)$ was set at $6 \mathrm{~mL} / \mathrm{kg}$, set ventilator frequency at 30 cycles $/ \mathrm{min}$, and PEEP at $5 \mathrm{~cm} \mathrm{H}_{2} \mathrm{O}$. Inspired oxygen was adjusted to keep the oxygen saturation between 91 and $95 \%$. Preterm infants born at $<30$ weeks gestation with respiratory distress routinely received caffeine shortly after birth. Surfactant was administered to preterm infants with RDS after intubation. Infants requiring rescue high-frequency oscillatory or jet ventilation were transitioned to a conventional ventilator before extubation for the pre-extubation peak $\mathrm{EA}_{\mathrm{di}}$ measurements. Only the first extubations in each infant were included in the analysis.

\section{QUICK LOOK}

\section{Current knowledge}

There is no objective tool to accurately predict extubation outcome. Successful extubation is dependent on effective respiratory drive and magnitude of respiratory load. $\mathrm{EA}_{\mathrm{di}}$ allows for quantification of neural respiratory drive in proportion to respiratory load and has been increasingly used in ventilator weaning and diagnostics.

\section{What this paper contributes to our knowledge}

This small study did not find $\mathrm{EA}_{\mathrm{di}}$ to be a predictor of extubation outcome in preterm neonates. However, role of $\mathrm{EA}_{\mathrm{di}}$ should be studied further to improve management of mechanically ventilated neonates.

A specialized feeding tube with an embedded electrode array $\left(\mathrm{EA}_{\mathrm{di}}\right.$ catheter, Maquet Solna, Sweden), instead of a standard nasogastric feeding tube, was inserted into the esophagus within a few hours of intubation and after obtaining parental consent. Data from the $\mathrm{EA}_{\mathrm{di}}$ catheter were utilized by Servo-i ventilator software for continuous $\mathrm{EA}_{\mathrm{di}}$ recording. Correct placement and positioning of the $\mathrm{EA}_{\mathrm{di}}$ catheter was verified using the retro-cardiac electrocardiograph waveform on the Servo-i ventilator monitor at the beginning of each shift and then intermittently thereafter. At other times, the Servo-I screen was covered so that the staff were not aware of continuous $\mathrm{EA}_{\mathrm{di}}$ measurements. Data, including peak $\mathrm{EA}_{\mathrm{di}}$ and minimum $\mathrm{EA}_{\mathrm{di}}$, were stored in 1-min intervals using Servo-i software and then downloaded every $24 \mathrm{~h}$ and transferred into Microsoft Excel. Stata (StataCorp, College Station, Texas) was used for statistical analysis.

We collected information on infant demographics, set respiratory variables $\left(\mathrm{F}_{\mathrm{IO}_{2}}\right.$, targeted $\mathrm{V}_{\mathrm{T}}$, PEEP, and ventilator frequency) and measured respiratory variables (mean airway pressure $\left[\overline{\mathrm{P}}_{\mathrm{aw}}\right]$ and peak inspiratory pressure [PIP]) from the electronic medical record flow sheet every $6 \mathrm{~h}$ for $24 \mathrm{~h}$ before extubation. We used peak $\mathrm{EA}_{\mathrm{di}}$ as a measure of inspiratory drive and calculated the $\mathrm{V}_{\mathrm{T}}$ /peak $\mathrm{EA}_{\mathrm{di}}$ ratio to quantify the subject's effort per $\mathrm{V}_{\mathrm{T}}$. A modified respiratory severity index was calculated for each infant at the time of intubation $\left(\overline{\mathrm{P}}_{\mathrm{aw}} \times \mathrm{F}_{\mathrm{IO}_{2}}\right) \cdot{ }^{18}$ All measurements took place in the NICU while the infants were receiving routine care. If an infant required high-frequency ventilation, data were obtained only after transition to a conventional ventilator, at least $6 \mathrm{~h}$ before extubation.

The clinical care team made the decision to extubate once the infants met clinical extubation criteria, which included clinical stability, a low $\mathrm{F}_{\mathrm{IO}_{2}}(<0.40)$, a low $\overline{\mathrm{P}}_{\text {aw }}$ $(8-10 \mathrm{~mm} \mathrm{Hg})$ on a set $\mathrm{V}_{\mathrm{T}}$, and an acceptable blood gas ( $\mathrm{pH}>7.25$ and $\mathrm{P}_{\mathrm{CO}_{2}} 45-55 \mathrm{~mm} \mathrm{Hg}$ ). All infants were 
Table 1. Infant Characteristics by Extubation Outcome

\begin{tabular}{|c|c|c|c|}
\hline Characteristics & Successful Extubation & Failed Extubation & $P$ \\
\hline Subjects, $n$ & 14 & 7 & \\
\hline Gestational age, mean $\pm \mathrm{SD}$ week & $29 \pm 2.6$ & $26.7 \pm 1.8$ & .057 \\
\hline Birthweight, mean $\pm \mathrm{SD} g$ & $1,417 \pm 632$ & $788 \pm 266$ & .02 \\
\hline Apgar score, median & 8 & 6 & .08 \\
\hline Intubation in delivery room, $\%$ & 35 & 71 & .19 \\
\hline Modified respiratory severity index at intubation $\left(\mathrm{P}_{\mathrm{aw}} \times \mathrm{F}_{\mathrm{IO}_{2}}\right)$, mean $\pm \mathrm{SD}$ & $4.15 \pm 4.2$ & $3.05 \pm 1.6$ & .54 \\
\hline
\end{tabular}

$\overline{\mathrm{P}_{\mathrm{aw}}=\text { mean airway pressure }}$

extubated to noninvasive ventilation, which included nasal intermittent positive-pressure ventilation or bubble CPAP, and continued until ready to wean off to room air.

During the course of the study period, INSURE (intubatesurfactant-extubate) ${ }^{19}$ was implemented to facilitate early extubation in preterm infants requiring intubation for the surfactant administration only. These infants were intubated briefly and extubated within a few hours without going through the weaning process. We also included the pre-extubation peak $\mathrm{EA}_{\mathrm{di}}$ data from these infants for the analysis.

Statistical Analyses. We considered extubation successful if the infant did not require mechanical ventilation for at least $72 \mathrm{~h}$ after extubation. We compared the mean peak EA $_{\mathrm{di}}$ measures between infants in whom extubation failed or succeeded using the Student $t$ test with a significance level of $P<.05$ and using mixed-effects regression analysis for repeated measurements. We used Stata IC/12 software for all statistical analyses.

\section{Results}

All infants were initially stabilized receiving CPAP in the delivery room and received surfactant after intubation for RDS. Three infants received surfactant as per the INSURE protocol and were extubated rapidly without weaning. Of the 21 participants, $67 \%$ were male. Fortyeight percent of the infants were intubated in the delivery room, all received surfactant, and $81 \%$ received caffeine. The mean \pm SD gestational age and birthweight were $28 \pm 2.6$ weeks and $1,208 \pm 611 \mathrm{~g}$, respectively.

All infants met clinical and biochemical criteria for extubation as described above. Two thirds of the infants $(n=14)$ experienced a successful first extubation, whereas one third failed $(n=7)$. Infants with failed extubations were smaller and of lower gestational age compared with those with successful extubation (Table 1). The failed extubation group was also more likely to be intubated in the delivery room compared with those with successful extubations despite comparable respiratory severity at the time of intubation.

The median time to first extubation attempt was $1 \mathrm{~d}$ (interquartile range 1-2 d) and was not different between the successful and failed extubation groups. The respiratory and ventilator variables ( $\overline{\mathrm{P}}_{\mathrm{aw}}$, PIP, PEEP, set ventilator frequency, $\mathrm{F}_{\mathrm{IO}_{2}}, \mathrm{~V}_{\mathrm{T}} / \mathrm{kg}$, and breathing frequency) and $\mathrm{EA}_{\mathrm{di}}$-derived parameters (peak $\mathrm{EA}_{\mathrm{di}}$ and the ratio of $\mathrm{V}_{\mathrm{T}}$ per $\mathrm{kg}$ to peak $\mathrm{EA}_{\mathrm{di}}$ ) were also not different between the 2 groups $1 \mathrm{~h}$ before extubation (Table 2).

There was considerable variability in the measured peak $\mathrm{EA}_{\mathrm{di}}$ level within and between subjects during mechanical ventilation. There was no correlation between birthweight or gestational age and pre-extubation peak $\mathrm{EA}_{\mathrm{di}}$. The peak $\mathrm{EA}_{\mathrm{di}}$ trend $1 \mathrm{~h}$ before extubation was not different between the 2 groups (Fig. 1). The mean peak $\mathrm{EA}_{\mathrm{di}}$ measured at 1 , 6 , and $24 \mathrm{~h}$ before extubation were also similar between the successful and failed extubation groups. Exclusion of data from infants extubated with the INSURE protocol did not alter the result.

\section{Discussion}

This study shows that peak $\mathrm{EA}_{\mathrm{di}}$ and other $\mathrm{EA}_{\mathrm{di}}$-derived parameters did not differ between preterm infants with successful and failed extubations. There was, however, a strong association between both birthweight and gestational age and the extubation outcome, with the smallest and most immature infants having a lower extubation success rate.

The most straightforward explanation of our findings is that peak $\mathrm{EA}_{\mathrm{di}}$ during the period before extubation does not predict a successful extubation in preterm infants. There are other explanations that could account for our failure to observe significant differences in peak $\mathrm{EA}_{\mathrm{di}}$ in relation to extubation outcome. First, whereas prolonged mechanical ventilation would be expected to cause pronounced diaphragmatic dysfunction and increase the risk of extubation failure, ${ }^{9,20}$ most subjects were extubated in $<48 \mathrm{~h}$ before significant diaphragmatic dysfunction occurred. Second, ventilator assist during peak $\mathrm{EA}_{\mathrm{di}}$ measurement, although minimal in the pre-extubation period, might have affected the measured peak $\mathrm{EA}_{\mathrm{di}}$ levels. In addition, it is possible that mechanical ventilation in volume guarantee mode may have affected our results. Whereas the infant's breathing effort (and measured peak $\mathrm{EA}_{\mathrm{di}}$ ) reflects the ability to 


\section{EA $_{\text {DI }}$ IN Preterm Infants and Extubation Success}

Table 2. Pre-Extubation Ventilator Support and Electrical Activity of the Diaphragm Values in Successful and Failed Extubations

\begin{tabular}{|c|c|c|c|}
\hline Variables & Successful Extubation & Failed Extubation & $P$ \\
\hline Time to first extubation, median (IQR) d & $1(1-2)$ & $2(1-6)$ & .23 \\
\hline \multicolumn{4}{|l|}{ Pre-extubation ventilator support and respiratory variables } \\
\hline Measured $\overline{\mathrm{P}}_{\mathrm{aw}}$, mean $\pm \mathrm{SD} \mathrm{cmH}_{2} \mathrm{O}$ & $9.2 \pm 2.9$ & $8.3 \pm 1.4$ & .54 \\
\hline Measured PIP, mean $\pm \mathrm{SD} \mathrm{cmH}_{2} \mathrm{O}$ & $17.7 \pm 6.7$ & $18.6 \pm 3.9$ & .66 \\
\hline Set PEEP, mean $\pm \mathrm{SD} \mathrm{cmH}_{2} \mathrm{O}$ & $5.07 \pm 0.26$ & $5.3 \pm 0.4$ & .20 \\
\hline Set ventilator frequency, mean $\pm S D$ & $31 \pm 10$ & $26 \pm 11$ & .23 \\
\hline Set $\mathrm{V}_{\mathrm{T}}$, mean $\pm \mathrm{SD} \mathrm{mL} / \mathrm{kg}$ & $5.5 \pm 0.78$ & $5.3 \pm 0.79$ & .45 \\
\hline Set $\mathrm{F}_{\mathrm{IO}_{2}}$, mean $\pm \mathrm{SD}$ & $0.25 \pm 0.10$ & $0.25 \pm 0.06$ & .88 \\
\hline Measured breathing frequency, mean \pm SD breaths/min & $55 \pm 14$ & $55 \pm 15$ & .95 \\
\hline \multicolumn{4}{|c|}{ Pre-extubation peak $\mathrm{EA}_{\mathrm{di}}$ and peak $\mathrm{EA}_{\mathrm{di}}$-derived values, mean $\pm \mathrm{SD}$} \\
\hline \multicolumn{4}{|c|}{ Average pre-extubation peak $\mathrm{EA}_{\mathrm{di}}$, mean $\pm \mathrm{SD} \mu \mathrm{V}$} \\
\hline 1-h pre-extubation period & $10.36 \pm 4.89$ & $9.69 \pm 4.75$ & .66 \\
\hline 6-h pre-extubation period & $9.24 \pm 3.24$ & $10.21 \pm 4.04$ & .55 \\
\hline 24-h pre-extubation period & $7.95 \pm 1.64$ & $9.67 \pm 3.18$ & .63 \\
\hline $\mathrm{V}_{\mathrm{T}} / \mathrm{kg} /$ peak $\mathrm{EA}_{\mathrm{di}}$ ratio & $0.76 \pm 0.52$ & $0.6 \pm 0.27$ & .46 \\
\hline $\begin{array}{l}\mathrm{IQR}=\text { interquartile range } \\
\overline{\mathrm{P}}_{\mathrm{aw}}=\text { mean airway pressure } \\
\mathrm{PIP}=\text { peak inspiratory pressure } \\
\mathrm{V}_{\mathrm{T}}=\text { tidal volume } \\
\mathrm{EA}_{\mathrm{di}}=\text { electrical activity of the diaphragm }\end{array}$ & & & \\
\hline
\end{tabular}

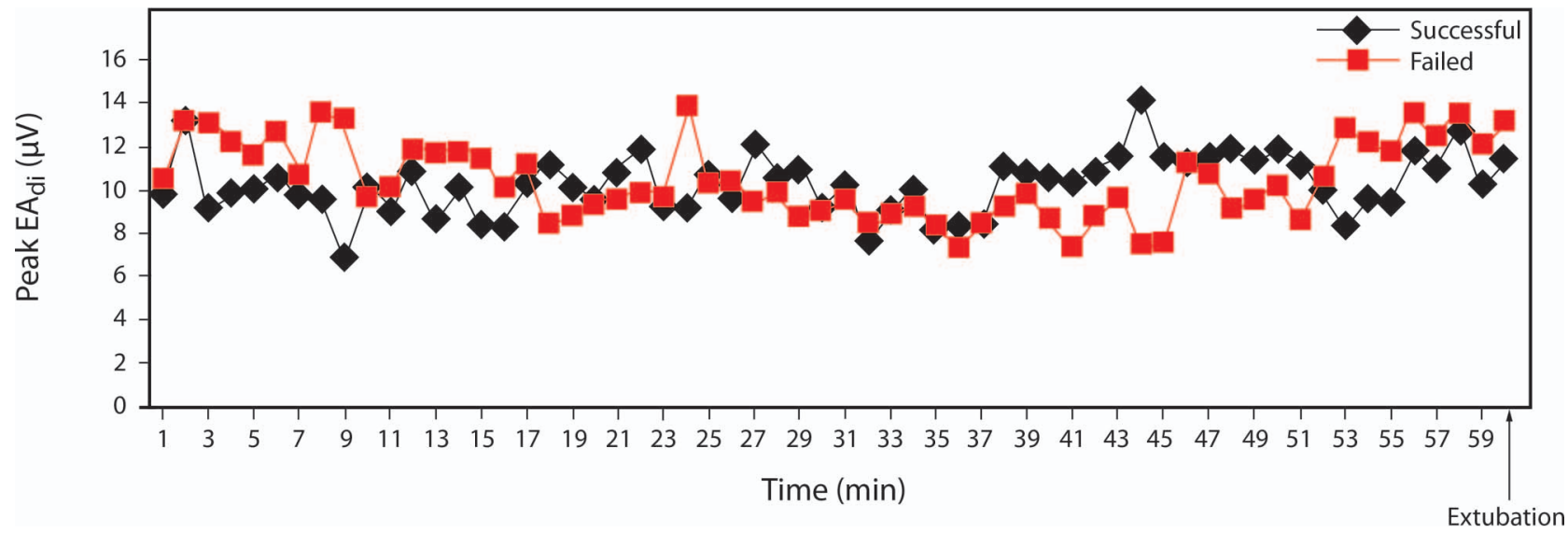

Fig. 1. Peak $E A_{d i} 1 \mathrm{~h}$ before extubation in subjects with successful and failed extubations.

generate adequate inspiratory effort in proportion to the respiratory load, it also reflects the appropriateness of the set $\mathrm{V}_{\mathrm{T}}$ target. If the targeted $\mathrm{V}_{\mathrm{T}}$ is set too low, the inspiratory work load is shifted from the ventilator to the patient, resulting in higher observed peak $\mathrm{EA}_{\mathrm{di}}$ and an increase in breathing frequency to optimize the minute ventilation (which, in turn, lowers the measured PIP and $\overline{\mathrm{P}}_{\mathrm{aw}}$ ). However, as indicated in Table 2, we observed similar $\mathrm{V}_{\mathrm{T}}$ levels (adjusted per $\mathrm{kg}$ ), breathing frequencies, and peak $\mathrm{EA}_{\mathrm{di}}$ in the 2 groups before extubation, suggesting equivalent levels of ventilator assist in both groups. We also noted higher PIPs and a lower $\overline{\mathrm{P}}_{\mathrm{aw}}$ (although not statistically different) in the extubation failure group. This opposite trend in PIP and $\overline{\mathrm{P}}_{\mathrm{aw}}$ may have been secondary to the inaccuracies introduced by reporting data that were intermittently collected and manually entered into the electronic medical record.

Although $\mathrm{EA}_{\mathrm{di}}$ has been successfully used in providing neurally adjusted ventilatory assist and in ventilator weaning and diagnostics, only few studies have evaluated this innovative technology to predict extubation outcome. Wolf et $\mathrm{al}^{10}$ measured $\mathrm{EA}_{\mathrm{di}}$ in older children $(4 \mathrm{~d}$ to $18 \mathrm{y})$ with heterogeneous lung disease before and during an extubation readiness test while they were receiving minimum pressure support ventilation. Similar to our study, no difference was observed in the $\mathrm{EA}_{\mathrm{di}}$ between subjects who passed or failed an extubation readiness test. However, they reported a significant difference in the ratio of $\mathrm{V}_{\mathrm{T}}$ to 


\section{EA $_{\text {DI }}$ IN Preterm Infants and Extubation Success}

EA $_{\mathrm{di}}$ between the 2 groups during the extubation readiness test, but not before the extubation readiness test when children were receiving pressure controlled ventilation. Liu et al ${ }^{15}$ evaluated EA $_{\mathrm{di}}$ during a spontaneous breathing test (SBT) without pressure support in adult subjects with COPD. They reported a significant increase in $\mathrm{EA}_{\mathrm{di}}$ during the SBT in the extubation failure group compared with those with extubation success, but again no difference was noted when receiving ventilator assist. Barwing et al ${ }^{17}$ similarly reported an early increase in $\mathrm{EA}_{\mathrm{di}}$ during an SBT performed after disconnection from a ventilator in the failed group compared with the successful group in critically ill and difficult-to-wean subjects. ${ }^{17}$ These studies suggest that $\mathrm{EA}_{\mathrm{di}}$ or $\mathrm{EA}_{\mathrm{di}}$-derived parameters such as the $\mathrm{V}_{\mathrm{T}} / \mathrm{EA}_{\mathrm{di}}$ ratio may predict extubation outcome when measured during an extubation readiness test or SBT. This also underscores the effect of any ventilator assist on the neural respiratory drive and, hence, on measured $\mathrm{EA}_{\mathrm{di}}$.

Our study differed from the above mentioned studies in a number of ways. We measured peak $\mathrm{EA}_{\mathrm{di}}$ in preterm neonates who have immature respiratory control, probably accounting for high variability in peak $\mathrm{EA}_{\mathrm{di}}$ during the study period. Also, we recorded peak $\mathrm{EA}_{\mathrm{di}}$ during ventilator assist and not during an SBT or extubation readiness test. There is only limited evidence supporting SBT for extubation readiness in the neonatal population, and it is not a standard practice in most neonatal units. ${ }^{4}$ In our nursery, we wean the ventilator support to a minimum before attempting extubation based on consensus-based guidelines. Other limitations of our study were a small sample size, an observational study design from a single center, and a unique preterm neonatal patient population with immature control of breathing. In addition, measured respiratory variables were obtained from data manually entered by the clinician into the electronic medical record and thus may not accurately reflect the mean of continuous measurements over time.

\section{Conclusions}

Although a promising respiratory vital sign, we did not find absolute peak $\mathrm{EA}_{\mathrm{di}}$ or $\mathrm{EA}_{\mathrm{di}}$-derived parameters helpful to predict extubation outcome in preterm neonates. $\mathrm{EA}_{\mathrm{di}}$ may be useful in determining extubation readiness with an SBT in a subset of patients, such as difficult-towean infants or those with diaphragmatic dysfunction from prolonged mechanical ventilation.

\section{ACKNOWLEDGMENTS}

We acknowledge the nurses and respiratory therapists at The Children's Hospital at Dartmouth for help during the study. We extend special recognition to Dr Robert Drake for support in writing and editing of the manuscript. We also thank Maquet (Solna, Sweden) for technical support and supplies (software for data recording) during this study.

\section{REFERENCES}

1. Jobe AH, Ikegami M. Mechanisms initiating lung injury in the preterm. Early Hum Dev 1998;53(1):81-94.

2. Van Marter LJ, Allred EN, Pagano M, Sanocka U, Parad R, Moore $\mathrm{M}$, et al. Do clinical markers of barotrauma and oxygen toxicity explain interhospital variation in rates of chronic lung disease? The Neonatology Committee for the Developmental Network. Pediatrics 2000;105(6):1194-1201.

3. Newth CJ, Venkataraman S, Willson DF, Meert KL, Harrison R, Dean JM, et al. Weaning and extubation readiness in pediatric patients. Pediatr Crit Care Med 2009;10(1):1-11.

4. Kamlin CO, Davis PG, Morley CJ. Predicting successful extubation of very low birthweight infants. Arch Dis Child Fetal Neonatal Ed 2006;91(3):F180-F183

5. Mueller M, Wagner CL, Annibale DJ, Knapp RG, Hulsey TC, Almeida JS. Parameter selection for and implementation of a web-based decision-support tool to predict extubation outcome in premature infants. BMC Med Inform Decis Mak 2006;6:11.

6. Szymankiewicz M, Vidyasagar D, Gadzinowski J. Predictors of successful extubation of preterm low-birth-weight infants with respiratory distress syndrome. Pediatr Crit Care Med 2005;6(1):44-49.

7. Gillespie LM, White SD, Sinha SK, Donn SM. Usefulness of the minute ventilation test in predicting successful extubation in newborn infants: a randomized controlled trial. J Perinatol 2003;23(3):205-207.

8. Dimitriou G, Greenough A, Endo A, Cherian S, Rafferty GF. Prediction of extubation failure in preterm infants. Arch Dis Child Fetal Neonatal Ed 2002;86(1):F32-F35.

9. Levine S, Nguyen T, Taylor N, Friscia ME, Budak MT, Rothenberg $\mathrm{P}$, et al. Rapid disuse atrophy of diaphragm fibers in mechanically ventilated humans. N Engl J Med 2008;358(13):1327-1335.

10. Wolf GK, Walsh BK, Green ML, Arnold JH. Electrical activity of the diaphragm during extubation readiness testing in critically ill children. Pediatr Crit Care Med 2011;12(6):e220-e224.

11. Jubran A, Grant BJ, Laghi F, Parthasarathy S, Tobin MJ. Weaning prediction: esophageal pressure monitoring complements readiness testing. Am J Respir Crit Care Med 2005;171(11):1252-1259.

12. Sinderby C, Navalesi P, Beck J, Skrobik Y, Comtois N, Friberg S, et al. Neural control of mechanical ventilation in respiratory failure. Nat Med 1999;5(12):1433-1436.

13. Pang PC, Pepper MG, Ducker DA. Monitoring respiratory activity in neonates using diaphragmatic electromyograph. Med Biol Eng Comput 1995;33(3 Spec No):385-390.

14. Stein H, Firestone K, Rimensberger PC. Synchronized mechanical ventilation using electrical activity of the diaphragm in neonates. Clin Perinatol 2012;39(3):525-542.

15. Liu L, Liu H, Yang Y, Huang Y, Liu S, Beck J, et al. Neuroventilatory efficiency and extubation readiness in critically ill patients. Crit Care 2012;16(4):R143.

16. Luo YM, Hart N, Mustfa N, Lyall RA, Polkey MI, Moxham J. Effect of diaphragm fatigue on neural respiratory drive. J Appl Physiol 2001;90(5):1691-1699.

17. Barwing J, Pedroni C, Olgemöller U, Quintel M, Moerer O. Electrical activity of the diaphragm $\left(\mathrm{EA}_{\mathrm{di}}\right)$ as a monitoring parameter in difficult weaning from respirator: a pilot study. Crit Care 2013;17(4):R182.

18. Iyer NP, Mhanna MJ. Non-invasively derived respiratory severity score and oxygenation index in ventilated newborn infants. Pediatr Pulmonol 2013;48(4):364-369.

19. Dani C, Corsini I, Bertini G, Fontanelli G, Pratesi S, Rubaltelli FF. The INSURE method in preterm infants of less than 30 weeks' gestation. J Matern Fetal Neonatal Med 2010;23(9):1024-1029.

20. Jaber S, Petrof BJ, Jung B, Chanques G, Berthet JP, Rabuel C, et al. Rapidly progressive diaphragmatic weakness and injury during mechanical ventilation in humans. Am J Respir Crit Care Med 2011; 183(3):364-371. 